\title{
La matematización de los procesos químicos. Primera parte
}

Artículo recibido: 13-03-2010 | Artículo aprobado: 11-06-2010

The Mathematization of Chemical Processes. Part One

\section{Rómulo Gallego Badillo*}

Royman Pérez Miranda**

Roberto Figueroa Molina*
Resumen: Dada la extensión del presente aporte se ha decidido dividirlo en dos partes. En la primera se adelanta una reconstrucción de la historia del modelo cinético molecular de los gases y de la termodinámica clásica, y se realiza una aproximación al análisis epistemológico. En la segunda se intenta también una reconstrucción histórica de la creación de la fisicoquímica como disciplina científica y la introducción de la mirada desde la termodinámica clásica de los procesos químicos, asumidos como sistemas y como "máquinas térmicas". Se irá más allá de la inicialmente designada como "dinámica química". El objetivo consiste en elaborar una explicación admisible que dé cuenta de la introducción en el discurso químico, de una matemática de mayor complejidad que la propia del álgebra, dentro de la cual se construyó, durante la segunda mitad del siglo XIX, la química como ciencia, construcción ésta basada en las leyes ponderales.

Palabras clave: historia, epistemología, modelo cinético molecular de los gases, termodinámica clásica.

\begin{abstract}
Given the scope of this contribution the authors have decided to divide it in two parts. In the first, they develop a history reconstruction of the Molecular-Kinetic Model of Gases and the Classical Thermodynamics, and an approach to the epistemological analysis. In the second, there is an attempt to get a historical reconstruction of the creation of the Physical Chemistry as a scientific discipline and the introduction from the perspective of the classical thermodynamics of the chemical processes, given as systems and "heat engines." It is intended go beyond the initial task designated as "Dynamic Chemistry".

The study is aimed at elaborating an acceptable explanation in the chemistry discourse which shows the introduction of a more complex Mathematics than the one of algebra where Chemistry as a science was built during the second middle of the Nineteenth century.
\end{abstract}

Keywords: History, Epistemology, Philosophy, Kinetic Molecular model of Gases, Classical Thermodynamics.

* Profesor de la Universidad Pedagógica Nacional. Bogotá, D. C. Colombia. rgallego@pedagogica.edu.co

* Profesor de la Universidad Pedagógica Nacional. Bogotá, D. C. Colombia. royman@pedagogica.edu.co.

** Grupo de Investigación Representaciones y Conceptos Científicos - Grupo IREC Profesor del Doctorado en Educación, RUDECOLOMBIA. Universidad del Atlántico. Barranquilla. Colombia. robertfigue@hotmail.com 
La matematización de los procesos químicos: primera parte Leonor Camargo | Patricia Perry | Carmen Samper | Óscar Molina | Armando Echeverry

\section{Introducción}

Algunas reconstrucciones históricas afirman que, para entender el modelo atómico de J. Dalton (1766 - 1844) y la química de A. L. Lavoisier (1743 - 1794), no se requiere del dominio de una matemática avanzada (Schneer, 1975). Algo análogo habría que sostener en relación con las llamadas leyes ponderales con las que se construyó la química a lo largo del siglo XIX, que requieren de la aplicación de la regla de tres simple. El interés por darle sustento matemático al conocimiento químico, viene del trabajo adelantado por el discípulo de I. Kant (1724 - 1804), J. B. Richter (1762 - 1807). Ese interés lo plasmó en su obra "Principios de estequiometría o ciencia de medir los elementos químicos", que vio la luz en tres tomos, entre 1792 y 1793 (Lockemann, 1960). No obstante, ese interés de matematizar la química no pasará del nivel ya anotado. Con la adopción del modelo de Dalton y las leyes de la composición constante y de las proporciones múltiples, por parte de J. J. Berzelius (1779 - 1848), la comunidad de practicantes se dividirá entre atomistas y equivalentistas.

Son explicables las razones por las cuales en el primer programa académico para la formación profesional de los químicos, en la década de 1830, en la ciudad alemana de Giessen, en el de estudio la formación matemática parece que no se le confirió la importancia debida. Quizás como influencia de F. Stromeyer (1776 - 1835), el primero que en 1805 introdujo la "enseñanza práctica en el laboratorio", los químicos comenzaron a sostener que su ciencia era eminentemente experimental. El programa académico de J. von Liebig (1803 - 1873) fue de tal éxito, que se constituyó, con las adecuaciones requeridas, en el modelo para la formación de estos profesionales, tanto en Gran Bretaña como en los Estados Unidos de Norteamérica (Brock, 1998).
Ese dominio de la concepción experimentalista y positivista del plan de estudio creado por J. von Liebig, hay que atribuírselo al desarrollo de la química orgánica, desde el punto de vista del análisis y de la síntesis de sustancias existentes en la naturaleza como de la de aquellas no naturales (Gallego Badillo, Pérez Miranda, Torres de Gallego y Gallego Torres, 2004), a la vez que de reconocidos investigadores galardonados con el Premio Nobel. Aún cuando quienes se han dedicado a la reconstrucción histórica del desarrollo de esta ciencia, suelen atribuirle un papel secundario a la industrialización, es menester subrayar que es ella, con la de los colorantes y la de productos farmacéuticos, es esta industrialización la que definitivamente fortalece la concepción, el modo de pensar y de producir de los químicos orgánicos. Señálese que con base en las leyes ponderales y el modelo atómico de J. Dalton (1766 - 1844) admitidos por J. J. Berzelius (1779 - 1848), se hizo posible la construcción de la química como ciencia, en el campo de la química orgánica. Los resultados de las investigaciones hicieron necesario que paulatinamente el modelo atómico de Dalton fuera transformado hasta su abandonado definitivo al final del siglo XIX. En estricto rigor, no se puede hablar del triunfo de los atomistas sobre los equivalentistas.

Por fuera de los reconocidos centros universitarios alemanes en los que los químicos orgánicos eran la referencia, al margen de ellas emergerán tres personajes que chocaran con esa versión experimentalista basada en una matemática relativamente elemental. Ellos serán J. E. van't Hoff (1852 - 1911), W. Ostwald (1853 - 1932) y S. Arrhenius (1859 - 1927). Los tres crearán la fisicoquímica convirtiendo los procesos químicos en sistemas termodinámicas y por tanto, introduciendo las ecuaciones diferenciales. Para apoyar esta nueva disciplina fundarán 
la revista "Zeitschrift für physikaliscue Chemie"; acto este que generará una animadversión por parte de los químicos orgánicos, quienes pondrán en tela de juicio los postulados fisicoquímicos, alegando que sus conclusiones carecían de bases experimentales (Brock, 1998). Los químicos orgánicos no estaban en condiciones de entender esos postulados, puesto que carecían del dominio matemático requerido (Shneer, 1975). La empresa de socializar (Bowler y Morus, 2005) esta disciplina será completada por Ostwald redactando el primer texto escolar (Laidler, 1995). La fisicoquímica será el puente conceptual y metodológico entre la física y la química, un hecho que contribuirá en el siglo XX una explicación "fisicalista" (Mayr, 2006) del estatuto epistemológico de esta ciencia, iniciada por G. Bachelard (1976) y continuada luego por W. B. Jensen (1998a; 1998b; 1998c).

\section{De los inicios}

Se podría considerar que los estudios acerca de la materialidad de la composición del mundo, tienen su origen en los procesos creados por los metalurgistas, con los que vino el convencimiento de que el ser humano se hallaba en capacidad de transformar esa composición. Las explicaciones que siguieron fueron las de los alquimistas; una ocupación original que los químicos hicieron de lado, por lo que nunca se sometieron a contrastaciones rigurosas las correspondientes afirmaciones sostenidas. Se deshicieron de una tradición que consideraron un lastre, en ese proceso que emprendieron los químicos alcanzar reconocimiento social (Bowler y Mourus, 2007) y convertir su ciencia en contenido académico (Bensaude - Vincent y Stenger, 1997; Brock, 1998). Hacer de sus elaboraciones ciencia escolar fue un paso indispensable, como se sabe, el reconocimiento es pleno a partir del momento en que cualquier saber científico se incorpora a los textos escolares (Sánchez, 2009). En lo que a la química se refiere, en esta sentido, se hizo un trabajo de recopilación (Gallego Torres y Gallego Badillo, 2009). Para cerrar este párrafo, se está de acuerdo con que lo que hoy se identifica como ciencia experimental, le debe mucho a las elaboraciones de los alquimistas artesanos (Fara, 2009), aquellos que inician la historia como alfareros y quienes desarrollan los hornos y los crisoles, sin los cuales la obtención del cobre metálico es inexplicable (Lévi-Strauss. 1964). Se hará metalurgista.

Hay que entender que para una comprensión de la alquimia se requieren precisiones históricas, con el fin de separar los alquimistas artesanos, de los místicos y de los charlatanes, estos últimos, los charlatanes, serán quienes generaron todo el rechazo y la persecusión oficial sin distinción alguna, sobre todos aquellos que se de declaran practicantes del arte alquímico $\mathrm{y}$, por su puesto as implicaciones religiosas consecuentes. La alquimia es relega al olvido con la publicación del libro "El químico escéptico" de R. Boyle.La construcción histórica de la química como ciencia, se remonta a la solución del problema técnico presentado por la bomba impelente - expelente de Ctesibio de Alejandría (Schneer, 1975). La solución creada por E. Torricelli (1608 - 1647) y V. Viviani (1622 - 1703), quienes inventaron el barómetro, el concepto de presión atmosférica. Demostraron además que el vacío era factible, el vacío barométrico. Que el aire pesaba, fue públicamente experimentado por Otto von Guericke (1602 - 1686). Los gases se hicieron objeto de estudio. R. Boyle (1627 - 1691) y R. Hooke (1635 - 1703) con sus experimentos darán origen a la "Química neumática" (Ihde, 1984).

R. Towneley (1629 - 1668) y H. Power (1623 - 1668) son quienes formulan por 
La matematización de los procesos químicos: primera parte Leonor Camargo | Patricia Perry | Carmen Samper | Óscar Molina | Armando Echeverry

primera vez la ley de las relaciones inversas entre la presión y el volumen. De ella se apoderó Boyle sin los reconocimientos debidos, en la segunda edición de su "New experiments physico - mechanical touching the Spring of the Air". E. Mariotte (1620 1684) en su libro "De la nature de I'air", también sin aludir a Boyle, dará a conocer como una elaboración suya, la misma ley (Laidler, 1995). Con estas anotaciones sobre el innegable plagio, dentro de una enseñanza de las ciencias que le da la espalda a la historia, los profesores la identifican como la ley de Boyle - Mariotte (Gallego Badillo, Gallego Torres y Pérez Miranda, 2006).

Una primera explicación de esta ley es la elaborada por D. Bernouilli (1700 1782), reconocido como matemático, que la dio a conocer en 1738 , en un capítulo de su "Hydrodynamica". El modelo icónico de la "mesa de billar" se convirtió en una referencia algunas veces no suficientemente analizado cuando la el modelo científico molecular de los gases, se hace objeto de trabajo en el aula o los profesores de ciencias son conminados a responder por y llenar esos formularios creados por los técnicos de los ministerios de educación. Sobre la formulación definitiva del modelo molecular de los gases, se discutirá en párrafos posteriores.

En cuanto a la relación volumen temperatura, está fue desarrollada realmente desarrollada por J. L. Gay-Lussac (1778 1850). Aun cuando en los países anglos se le conoce como ley de Charles, por J. A. C. Charles (1746 - 1823). Se afirma que nunca la publicó. Gay-Lussac le otorgó a Charles el debido reconocimiento, en la publicación en la que dio a conocer su ley, en 1802 (Laidler, 1995). Es la ley de Charles y GayLussac. Entre las muchas contribuciones del científico francés, además de su ley de los volúmenes de combinación, cuya suma aparentemente anómala, fue explicada por A. Avogadro (1776 - 1856), hay que destacar que fue él al mismo tiempo que J. Dalton (1766 - 1844), quienes en 1802 determinaron por primera vez el coeficiente de dilatación térmica de los gases, encontrando ambos el mismo valor, 1/266. Este valor fue muchos años después rectificado por $\mathrm{H}$. G. Magnus $(1802$ - 1870) y H. V. Regnault (1810 - 1878). Lo aceptado hoy es el valor de 1/273 (Lockemann, 1960). De ahí viene el conocido número "273", empleado para convertir la temperatura Celsius en temperatura absoluta.

A la relación matemática directa entre la variación del volumen con la temperatura, se haya ligado G. Amontons (1663 - 1705). Sin embargo y pocas veces no referenciado en los textos de enseñanza, parece ser quien formuló la relación directa entre el incremento de la presión de un gas, con el aumento de la temperatura. Con esta formulación de las denominadas "leyes empíricas del comportamiento de los gases", el cuadro para que entraran a ser el fundamento de la termodinámica clásica, se completó. A manera de paréntesis, es indispensable señalar que a partir de una reflexión histórica y epistemológica, se intentó demostrar que no había razones para seguir afirmando de estas leyes, que eran empíricas, como la mirada positivista suele considerarlas (Gallego Badillo, Pérez Miranda y Gallego Torres, 2009).

En el lenguaje actual la ley de Boyle y Mariotte se expresa, $\mathrm{PV}=\mathrm{k}_{1}$, para $\mathrm{N}$ y $\mathrm{T}$ constantes.

Donde $\mathrm{N}$ es la masa en términos de número de moles y $\mathrm{T}$ la temperatura absoluta, que no es una temperatura termométrica. Expresión esta que no recoge la dinámica de esa relación y que sirve para que el estudiantado resuelva los acostum- 
brados ejercicios de lápiz y papel. Puesto que se trata de la matematización de los procesos químicos, que aparece en todos los cuestionarios de las pruebas globalizadas.

Para la ley de Charles y Gay-Lussac, V $=\mathrm{k}_{2} \mathrm{~T}$, para $\mathrm{N}$ y P constantes. Así,

$\mathrm{dV}=-\mathrm{k}_{1}\left(\mathrm{dP} / \mathrm{P}^{2}\right), \quad(\mathrm{D} / \mathrm{PP})_{\mathrm{T}^{\prime} \mathrm{N}}=-\mathrm{k}_{1} / \mathrm{P}^{2}, \mathrm{dV}=$ $\mathrm{k}_{2} \mathrm{dT},(\partial \mathrm{V} / \partial \mathrm{T})_{\mathrm{PN}^{\prime}}=\mathrm{k}_{2}$

Si un sistema termodinámico gaseoso $\mathrm{S}$ se define analíticamente como

$\mathrm{S}=\mathrm{S}(\mathrm{P}, \mathrm{T})$, Entonces, $\mathrm{dV}=(\mathrm{DV} / \mathrm{PP})_{\mathrm{T}^{\prime} \mathrm{N}} \mathrm{dP}+$ $(\partial \mathrm{V} / \partial \mathrm{T})_{\mathrm{P}^{\prime} \mathrm{N}} \mathrm{dT}$

$d V=-k_{1} d P / P^{2}+k_{2} d T$

Puesto que, $\mathrm{k}_{1}=\mathrm{PV}$ y $\mathrm{k}_{2}=\mathrm{V} / \mathrm{T}$

$d V=-V d P / P+V d T / T ; \quad d V / V=-d P / P+d T / T$

Integración entre un volumen inicial $v_{i} y$ un volumen final $V_{f}$ una presión inicial $P_{i} y$ final $P_{f}$, dentro de las temperaturas correspondientes, $T_{i}$ y $T_{f}$, se llega a la ecuación de estado. Recordando que la integral $\mathrm{dx} / \mathrm{x}$ es Inx, se tiene que,

$\ln \left(V_{f} / V_{i}\right)=-\ln \left(P_{f} / P_{i}\right)+\ln \left(T_{f} / T_{i}\right)=\ln \left(P_{i} / P_{f}\right)\left(T_{f} / T_{i}\right)$

$V_{f} / V_{i}=P_{i} T_{f} / P_{f} T_{i}$ por lo que $\left(V_{f} / V_{i}\right)\left(P_{f} / P_{i}\right)=T_{f} / T_{i}$

Por transitividad,

$\mathrm{PV}=\mathrm{RT}$, para $\mathrm{N}=1$ y de manera general, $\mathrm{PV}=\mathrm{nRT}$

\section{El modelo cinético molecular de los gases}

Dentro de la categoría epistemológica de modelo científico (Lombardi, 1996; Del Re, 2000; Caldin, 2002), este modelo puede ser clasificado como analógico, ya que desde la primera formulación por parte de $\mathrm{D}$. Bernouilli en 1738 , se acudió a la representación de un artefacto idealizado, la mesa de billar. Es el comportamiento elástico de los gases el que posibilita la conversión de la categoría filosófica de átomo en un concepto científico. En este proceso interviene I. Newton (1643 - 1727), en quien se apoyó J. Dalton para formular el primer modelo químico. Destáquese que Dalton era meteorólogo de formación. Hay que recordar que con el experimento de los hemisferios de Magdeburgo, preparado y conducido por O. von Guericke (1602 - 1686), quedó definitivamente establecido que el aire pesa, tiene masa. Se requerirá, por supuesto, de la aceptación por parte de la comunidad de especialistas.

Contribuyó en este propósito J. Herapath (1790 - 1868). Aún cuando sostuvo que el movimiento de las partículas de un gas confinado en un recipiente estaba relacionado con el calor y con la presión, el tratamiento que llevó a cabo sobre las colisiones elásticas entre estas no fue satisfactorio, además de que llegó a la conclusión de que PV era proporcional a $\mathrm{T}^{2}$. El siguiente personaje que aportó a la construcción del modelo cinético molecular, fue J. J. Waterston (1811 - 1883). En un artículo que remitió a la "Philosophical Transactions" en 1846, incluyó por primera vez en la historia un tratamiento de la distribución de la energía entre moléculas de diferentes masas, propuso que la velocidad de estas era proporcional a la raíz cuadrada de la temperatura y suministró una admisible conceptualización de los calores específicos. Su artículo fue publicado nueve años después de su fallecimiento y en 1892, redescubierto en los archivos de la "Philosophical Transactions" y publicado (Laidler, 1995).

De acuerdo con K. J. Laidler (1995), en 1856 A. K. Krönig (1822 - 1879), publicó una corta memoria acerca del modelo ciné- 
tico molecular que, en principio, no tuvo gran significado. No obstante fue inspiradora para las contribuciones definitivas de R. Clausius (1822 - 1888) y de J. C. Maxwell (1831 - 1879). Clausius derivó la ecuación fundamental que relaciona $\mathrm{PV}$ con la masa $\mathrm{m}$ de una molécula, el número $\mathrm{N}$ y el cuadrado de la velocidad media. De esta manera, se estableció que $\mathrm{PV}=(1 / 3) \mathrm{Nmv}^{2}$. Puesto que la energía de traslación total es $\mathrm{E}_{\mathrm{k}}$ es $3 / 2 \mathrm{PV}$, Clausius dedujo que la diferencia entre la capacidad calorífica a presión constante $C_{p} y$ la capacidad calorífica a volumen constante $C_{v}$ y que a volumen constante es $\mathrm{PV} / \mathrm{T}$, por lo que $\mathrm{Q}$, el calor total en el gas está dado por,

$\mathrm{Q}=\mathrm{C}_{\mathrm{V}} \mathrm{T}=\left(\mathrm{C}_{\mathrm{V}} \mathrm{PV}\right) /\left(\mathrm{C}_{\mathrm{P}}-\mathrm{C}_{\mathrm{v}}\right)$

Cuando se examina el contenido de algunos textos didácticos universitarios, que tratan de aproximar a los estudiantes a los significados de $C_{v}$ y de $C_{p}$ por lo general, parecen dejar en ellos sentado la concepción sustancialista y anacrónica del calor, por cuanto la denominación, sin mayores explicaciones modernas de estos conceptos, conducen a una tergiversada concepción, en la que los sistemas termodinámicos poseen una "capacidad" de contener calor, como si esta función fuera función de estado. Al respecto, esa mirada anacrónica está precedida históricamente por la delimitación del "calor específico" que como quienes han tenido una aproximación al respecto, suelen repetir la expresión de que "calor perdido es equivalente a calor ganado". Se halla y se transmite por fuera del proceso que fue la construcción de la termodinámica clásica.

\section{Las conceptualziaciones de la presión y de la temperatura}

Una revisión de los textos de enseñanza acerca de la didactización del modelo cinético molecular de los gases, da pie para concluir que no se suele clarificar en los ejercicios de lápiz y papel, la diferencia entre la presión ejercida por el gas y la presión contra este, que produce compresión o expansión. La versión ahistórica que suelen transmitir, dejan de lado que para la conceptualización de la presión de un gas fue indispensable confinarlo en un recipiente cerrado. La distinción entre la presión interna de un gas y la que se ejerce sobre dicho gas la presión externa, es indispensable para una aproximación al concepto de trabajo mecánico. Desde la formulación de la existencia de la presión atmosférica y su medida con el barómetro, los desarrollos conceptuales y metodológicos posteriores de los trabajos con gases confinados, hicieron necesario el diseño y fabricación de manómetros. De la misma manera como el barómetro es en sí presión atmosférica, los manómetros son en general, presión. Por tanto, no solo son tecnológicos sino teóricos.

En lo tocante al concepto de temperatura y dentro de la concepción particularista dada por el modelo cinético molecular, como quedó establecido a finales del siglo XIX, esta dejó de ser la medida del calor, para hacer referencia, como ya se expresó, a la energía cinética promedio de las partículas que conforman un sistema termodinámico. Los termómetros basados en la dilatación de sustancias, en especial del mercurio, responden al principio termodinámico de que un aumento o disminución de la temperatura, causados por una diferencia de potencial térmico, se traduce en una dilatación o en una contracción de la sustancia termométrica empleada.

\section{La termodinámica clásica}

El término termodinámica fue empleado por primera vez, por W. Thomson, más tarde Lord Kelvin, en una serie de artícu- 
los que publicó entre 1851 y 1855, titulada "On the dynamical theory of heat" (Sobre la teoría dinámica del calor). Hay además que recordar que el fuego fue el principal factor interviniente en los procedimientos metalúrgicos de transformación. Luego lo será la combustión cuya explicación por parte de G. E. Stahl (1660 - 1734) que se conoce en la historia como "La teoría del flogisto", que propuso a partir de la idea de J. J. Becher (1635 - 1682) de las tres tierras $y$, particularmente de la "tierra grasa". Esta teoría, considerada por algunos investigadores como consistente y elegante (Izquierdo, 1986), fue adoptada por reconocidos químicos ingleses, entre los que cabe mencionar a H. Cavendish (1731 - 1810) y a J. Priestley (1733 - 1804), sin desconocer a C. W. Scheele (1742 - 1786). No obstante, y por problemas que no se entrarán a discutir, fue remplazada por la de la oxidación de A. L. Lavoisier. Esta teoría quedó para la posteridad como parte de la historia de la química (Bowler y Morus, 2007) y solo trabajada por especialistas interesados en la reconstrucción histórica de la constitución de la química como ciencia.

Es preciso considerar al respecto, la labor del profesor J. Black (1728 - 1799), quien fue uno de los primeros en renunciar a la teoría del flogisto y adoptar la de Lavoisier. Black es quien define la relación entre calor y temperatura, desde una perspectiva sustancialista, la temperatura como una medida del calor. Dentro de esta perspectiva conceptual, formuló el concepto de calor latente. Por otro lado, C. H. A. Holtzmann (1811 - 1865), sostuvo que un aumento en la temperatura incrementaba la elasticidad del gas y haría que ese gas produjera un trabajo mecánico que sería equivalente a ese aumento (Schneer, 1975). Las precisiones devendrían del diseño y construcción, primero, de las máquinas atmosféricas $y_{\text {, }}$ después, de las térmicas.
J. Watt (1736 - 1819), quien como encargado de la instrumentación científica y bajo la dirección de J. Black, diseñó la primera máquina de vapor. D. Papin (1647 - 1712) llegó como refugiado de las persecuciones contra los hugonotes y fue acogido por R. Hooke. De su análisis de los trabajos con la presión atmosférica, sugirió que esta podría ser empleada para la producción de trabajo mecánico. Se ligó con esto a una solución diferente del problema de la máquina impelente-expelente de Ctesibio de Alejandría. Esta idea se filtró, hasta el punto de que T. Savery (1650 - 1715) patentó el diseño que se ha reconocido como la máquina atmosférica (Ferguson, 1981). Los problemas técnicos y prácticos que tuvo esta máquina condujeron al rediseño y desplazamiento por la de T. Newcomen (1663 - 1729).

En la universidad de Glasgow existían como objeto de estudios modelos de la máquina de de Newcomen. Por alguna circunstancia, sufrieron desperfectos. Para su reparación fue encargado a Watt. Más allá de asumirse como un simple "mecánico", encaró su labor desde la concepción de Black del calor y emprendió un rediseño total de la misma que terminó en el diseño de la máquina de vapor. Además de formular el concepto de potencia ( $\mathrm{Hp})$, Watt se adentró en el cálculo de la eficiencia de su máquina, cuyos resultados no fueron positivos. S. Carnot (1796 - 1832), en 1824, en su "Réflexions sur la puissance motrice du feu et sur les machines propre á déveloper cette puissance" (Reflexiones acerca del poder mecánico del calor y de las máquinas diseñadas para desarrollar este poder), planteó este problema de una manera radicalmente distinta, para lo cual se ingenió una máquina ideal que opera en un ciclo perfectamente reversible conformado por cuatro etapas. Una expansión adiabática y otra isotérmica y una compresión adiabática y otra isotérmica. La máquina se encuentra 
entre dos manantiales, uno de alta y otro de baja temperatura. La analogía empleada por Carnot para el primer manantial fue la de un depósito de agua situado en lo alto. En esta representación la concepción de calor es sustancialista, es decir, los cuerpos contienen calor. Este trabajo significó un gran avance. Después, $B$. Thompson (1753 - 1814), quien adquirió el título de Conde de Rumford, un maestro de escuela que se casó con la viuda de Lavoisier, propuso la concepción mecánica del calor.

\section{El concepto de energía y de trabajo mecánico}

Según W. Berkson (1981) todas las expresiones referentes a la energía, están equivocadas y la única manera de aproximarse al concepto es mediante las matemáticas. No fue fácil. Su precisión solo se logró hacia la mitad del siglo XIX. Es este el espacio para resaltar que en algunos textos de enseñanza se suele enseñar que la energía es la "capacidad" que tiene un cuerpo para realizar trabajo. Al respecto, se podría suponer que detrás de esta definición didáctica, existiría una intencionalidad política y económica, con el fin de soslayar el contexto que posibilitó la construcción de la termodinámica clásica. Los historiadores resaltan que la construcción del concepto de energía, tiene una trayectoria de discusiones en torno a aquello que se quería significar con dicho término, en especial, por cuanto no era un observable (Bowler y Morus, 2005). Los seres humanos venían diseñando, construyendo y empleando la rueda hidráulica, para usos, por ejemplo, como el de los molinos; sin embargo este artefacto y sus aplicaciones, no condujo a pensar en este concepto. En efecto, la energía y mucho menos la ley de la conservación no pueden inferirse empíricamente. Al respecto cabe mencionar que algunos textos para la ense- ñanza de las ciencias en la secundaria, sostenían que "energía era la capacidad que tenía un cuerpo para realizar trabajo". Esta definición es en sí pobre, puesto que no despliega toda la complejidad del concepto, a la vez que no posibilita un pensamiento tecnológico para el diseño de tecnofactos, con miras a un desarrollo industrial.

Antes de la conceptualización actual, para referirse a este problema conceptual, se utilizaron las palabras "vis viva", luego del de "fuerza", como apareció en el libro de Sir W. R. Grove (1811 - 1896), de 1846, "Correlation of physical forces" (correlación de fuerzas), en el que habla del calor como una fuerza capaz de producir movimiento, hubo discusiones en torno a su significado. Un año después, H. L. F. von Helmholtz (1821 - 1894) publicó su artículo "Über Erhaltung der Kraft" (A cerca de la energía). La palabra alemana "Kraft" que algunos traducen como fuerza, realmente debe ser entendida como energía. El primero en distinguir conceptualmente la energía potencial fue W. J. M. Rankine (1820 - 1872), en su artículo de 1853 , "On the mechanical action of heat especially in gases and vapours"(Sobre el efecto mecánico del calor, especialmente en gases y vapores), que W. Thomson (1824 - 1907), bautizó como energía cinética. Esta se condensa en la fórmula, $E_{c}=1 / 2\left(m v^{2}\right)$. En todo caso, la construcción del concepto de conservación de la energía fue crucial para el despegue definitivo de la física. La energía es una función de estado, esto es, los sistemas contienen energía.

Algo semejante sucedió con el concepto de trabajo. Este fue inicialmente empleado por J. Watt, aun cuando no especificó a qué hacía referencia. Después de discusiones, fue delimitado con la contribución adelantada por C. A. Coulomb (1736 - 1806), en su ensayo "Théorie des machines simples" (Teoría de la máquinas simples), de 1781. Le 
seguirán las contribuciones de J. V. Poncelet (1788 - 1867). Cuando se examina la delimitación dada por la expresión dW = Fdx, donde " $x$ " es la longitud a lo largo de la cual se realiza el trabajo, muchas veces se olvida aclarar que $\mathrm{F}$ es una fuerza constante de oposición, contra la cual se hace el trabajo. Sucede algo análogo cuando se plantea el trabajo que se obtiene cuando un gas confinado en un cilindro se expande, elevando por ejemplo, una masa " $m$ ", colocada sobre el embolo. La presión no es la del gas, sino la ejercida por esa masa sobre dicho gas; la presión del gas es la que lleva a cabo el trabajo. En general, siempre el trabajo se lleva a cabo contra una fuerza opuesta. El trabajo no es una función de estado, sino de trayectoria, aparece en las fronteras del proceso. Los sistemas no contienen trabajo.

En cuanto a la ley de conservación de la energía, se suele citar al médico J. R. Mayer (1814 - 1878), quien intuyó que el calor y el trabajo eran formas de algo mucho más fundamental y convertibles entre sí. En la construcción del concepto de energía y de la ley de conservación de la misma, participó toda una pléyade de reconocidos científicos, quienes discutieron, en favor y en contra (Bowler y Morus, 2005), por lo que se puede afirmar que obedeció a un trabajo colectivo. J. P. Joule (1818- 1899) fue quien propuso una solución a la equivalencia entre calor y trabajo. Para el efecto acudió a los resultados de los experimentos electrolíticos para demostrar esa equivalencia. No obstante su propuesta no fue admitida en la revista de la "Royal Society". Su trabajo fue publicado hasta 1850 "On the mechanical equivalente of heat" (Sobre el equivalente mecánico del calor). En 1847, Joule asistió a una reunión de la "Asociación Británica para el Avance de la Ciencia" en la que expuso de manera breve las mediciones que había hecho sobre el "equivalente mecánico del calor". En ella estuvo presente el joven W. Thomson (1824
- 1907), Kelvin, quien se ocupaba del significado de la ley de la conservación y para quien el principio de Joule fue fundamental.

Kelvin se hallaba analizando la propuesta de Carnot en relación con las determinaciones de Joule. Encontró inicialmente que había una contradicción entre ambas propuestas. Esta contradicción la pone a circular en una serie de artículos publicados entre 1851 y 1855 , bajo el título genérico de "On the dynamical theory of heat, with numerical results deduced from Mr Joule's equivqlent of a termal unit, and M. Regnault's observations on steam" (Sobre la teoría dinámica del calor, desde los resultados numéricos deducidos por el señor Joule y las observaciones de M. Regnault). De conformidad con Carnot había un flujo de calor entre el manantial de alta al de baja temperatura. Esta era la diferencia de potencial térmico que, pasando por la máquina, generaba el trabajo mecánico. Según Joule, todo el calor debería convertirse en trabajo. Con anterioridad, Kelvin estableció la escala termodinámica de temperatura, que dio a conocer en "On an absolute thermometric scale founded on Carnot's theory of the motive power of heat, calculated from Regnault's observations" (En torno a una escala termométrica absoluta, basada en la teoría de Carnot sobre la potencia motriz del calor, calculada a partir de las observaciones de Regnault), de 1848. Hoy se ha acordado entre los especialistas, que no es una escala termométrica, que no depende de la dilatación de ninguna sustancia en particular. La dificultad técnica que en ese entonces significaba medir con precisión la cantidad de calor, le impidió a Kelvin en 1824, determinar que la cantidad de calor que era convertida en trabajo mecánico, era menor que la que procedía del manantial a alta temperatura.

Algunos historiadores son del parecer de que Kelvin formuló las leyes de la conser- 
vación de la energía y de la entropía (Bowler y Morus, 2005). No obstante, para inscribir en el proceso matemático de sus formulaciones específicas, es indispensable aludir a la discusión sobre la autoría del cálculo diferencial, entre Newton y G. W. Leibniz (1646 - 1716), cuya notación más asequible que la de Newton, terminó por imponerse en el continente europeo y condujo al desarrollo de las matemáticas, sobre todo en Alemania (Fara, 2009). En consecuencia, R. Clausius (1822 - 1888) se le adelantó en la solución, cuando en 1850, con su"Über die bewegende Kraft der Wärme und die Gesetze welche sich daraus für die Wärmelehere selbst ableiten lessen" (Sobre la energía en movimiento del calor y la ley por la cual ella es definible. Sus reflexiones verbales sobre este problema venían desde 1824, aún cuando es en este artículo en que esas consideraciones verbales son traducidas en ecuaciones diferenciales. Clausius sostuvo que no existía tal contradicción, puesto que en la producción de trabajo y al mismo tiempo, una cierta cantidad de calor se consume y otra se disipa (Schneer, 1975).

\section{La primera Ley}

Se había ya desarrollado el modelo cinético molecular. Desde la perspectiva del rechazo a la concepción sustancialista, $\mathrm{R}$. J. Clausius concluye que la energía del universo es constante. En 1850, en su "Über die bewegende Kraft der Wärme un die Gesetze welche sich deraus für die Wärmelehere selbst ableiten lessen", formula la primera ley de la termodinámica, que matemáticamente y en el lenguaje actual, se expresa mediante la ecuación,

$\mathrm{dE}=\mathrm{dQ}-\mathrm{dW} ; \quad \Delta \mathrm{E}=\mathrm{Q}-\mathrm{W}$

En la que $Q$ es el calor y W el trabajo mecánico equivalente.
La integral de $\mathrm{dE}$, entre el estado inicial y final del proceso es $\Delta E$, mientras que la de $\mathrm{dQ}$ y dW, son $\mathrm{Q}$ y W respectivamente, es decir, no es $\Delta \mathrm{Q}$ ni $\Delta \mathrm{W}$. Este delimitación matemática marca la diferencia entre una función de estado, como es el caso de $E$ y de una función de trayectoria. No hay un $\Delta \mathrm{Q}$ ni $\Delta \mathrm{W}$. Los sistemas termodinámicos no contienen $\mathrm{Q}$ ni W. La pregunta qué cantidad de calor o de trabajo contiene un sistema, carece de sentido.

\section{El concepto de entalpia}

La referencia inicial a este parámetro termodinámico fue hecha por J. W. Gibbs (1839 1903), quien se refirió a él como "contenido calorífico". En 1909, H. Kamerlingh Onnes (1853 - 1926), decidió darle el nombre actual de Entalpía (Laidler, 1995). Por tanto y de acuerdo con la primera ley,

$\mathrm{dH}=\mathrm{dE}+\mathrm{VdP}+\mathrm{PdV} ;$

a, $\mathrm{P}$ constante, $\mathrm{dH}=\mathrm{dE}+\mathrm{PdV}$,

En la que PdV es el trabajo a P constante. Así,

$d H=d Q_{p}$

Por lo que, $\mathrm{H}_{\mathrm{f}}-\mathrm{H}_{\mathrm{i}}=\Delta \mathrm{H}=\mathrm{Q}_{\mathrm{p}}$

La igualdad matemática no puede ser traducida aludiendo que la entalpías es calor. Ella significa que a presión constante el "flujo" de calor produce un cambio entálpico.

\section{La segunda Ley}

R. Clausius (1822 - 1888) resuelve la discusión existente al destacar que no todos los intercambios de calor se convierten en trabajo. Afirma que una parte del calor que "fluye" desde el manantial a alta temperatura al de baja temperatura se disipa y no 
puede emplearse para producir trabajo mecánico. Este racionamiento lo conduce a la necesidad de proponer una función que dé cuenta cuantitativamente de esa disipación. La denominará entropía y la representará con la letra "S". Esta propuesta la da a conocer, en 1865, en su "Über eine veränderte Form des zweiten Hauptsätzes der mechanischen Wärmetheorie" (Acerca de las diferentes maneras de expresar la segunda ley de la teoría mecánica del calor). Formula, entonces, la segunda ley como sigue,

$\mathrm{dS}=\mathrm{dQ}_{\mathrm{r}} / \mathrm{T}$

$\Delta S=Q_{r} / T \geq 0 ; T \Delta S=Q_{r}$

Esta ecuación y definición específica que se trata de un proceso reversible que se realiza a T constante. La conclusión de Clausius resalta que la entropía en el universo siempre tiende a crecer, es decir, mayor que cero; en otras palabras, que los procesos en el universo se realizan espontáneamente debido a esa tendencia. Cuando $\Delta \mathrm{S}$ es igual a cero, en principio, no sucede aparentemente nada significativo. Una vez formulado el concepto de entropía, se suscitaron discusiones filosóficas que sostuvieron la muerte técnica del universo.

Si se tiene en cuenta que esta magnitud no es un observable en sí misma, se comprende que su comprensión resulte inicialmente difícil de representar. Se dice que en una ágape de la alta sociedad inglesa de ese momento, un grupo de damas interesadas en la socialización de las ciencias, se acercó a Lord Kelvin interrogándolo sobre que era la entropía. Él las miró despectivamente y sin pensarlo dos veces afirmó que dijeran que era desorden (Thuillier, 1975). Una expresión casual que se propagó en los sistemas educativos a través de las transposiciones didácticas, que se ha perpetuado en la mayoría de los textos de enseñanza.
De una manera que peca de simplista y con propósitos didácticos, dejando de lado que la entropía es desorden, habría que insistir en la necesidad de una enseñanza de las ciencias que puntualice en que la producción y uso de la energía son costosos. Para solo acudir a un ejemplo, no toda la energía química liberada por combustión de los derivados del petróleo se convierte en el trabajo de impulsar a los automóviles. Una gran parte de ella se disipa en el aumento de la temperatura del motor. La disipación de esta energía en las máquinas térmicas y sus desarrollos posteriores, es uno de los problemas de los colectivos de tecnólogos. La formulación de las leyes de la termodinámica posee un contexto cultural, político y económico, que la enseñanza de la misma, abandonó.

\section{El significado estadístico de la entropía}

En uno de sus artículos de 1877, "Über die Bezihung zwischen den zweiten Hauptsatz der mechanischen Wämetheorie und den Wahrscheinlichkeitsrechnung respektive den Sätzen über des Wärmegleichgewicht", L. Boltzmann (1844 - 1906), desarrolló la concepción estadística de la entropía, condensada en la expresión,

$\mathrm{S}=\mathrm{k} \ln \Omega$

En esta "k" es la hoy denominada constante de Boltzmann y $\Omega$ define el número de las posibles configuraciones de las partículas en un estado dinámico dado del sistema. Delimita todas las posibilidades de encontrar esas partículas en los diferentes niveles de energía térmica probables de ese estado. Desde la estadística de Maxwell - Boltzmann, se puede calcular el nivel ocupado por el mayor número de esas partículas. Desde esta versión, la entropía es un concepto estadístico. 
La matematización de los procesos químicos: primera parte Leonor Camargo | Patricia Perry | Carmen Samper | Óscar Molina | Armando Echeverry

\section{Aspectos epistemológicos}

Muchos historiadores coinciden en afirmar que entre 1830 y 1840, A. Comte (1798 1857) desarrolló su propuesta filosófica identificada como positivismo. El positivismo tiene como columna vertebral la lógica inductiva. Esta concepción filosófica y epistemológica, afirman algunos especialistas, fue adoptada por quienes construyeron la termodinámica clásica. Imperaba igualmente entre la comunidad científica la admisión del determinismo (Laín y López, 1963). La elegancia matemática de la termodinámica clásica, su estructura lógica y sus resultados explicativos, sumados a que no se refería a ninguna sustancia en particular, generó la posición energetista, que defendía W. Ostwald y desde la que rechazaba el atomismo, como hipótesis innecesaria.

Es indispensable anotar que, examinada la historia de la construcción de la termodinámica clásica, su base en la observación de fenómenos de la naturaleza es muy poca. Excepto, por supuesto el hecho de la fabricación de cañones en Munich, por parte de Rumford y la determinación del equivalente mecánico del calor por Joule. Todo lo demás son deducciones basadas en el estudio de la máquina ideal de Carnot. Por otro lado, no fue fácil llegar al concepto de energía, que no es un observable en sí y que solo se llega a él después de analizar sus efectos, previa conceptualización del trabajo y del calor. La entalpía y la entropía, tampoco son observables. En consecuencia, no se llegó a la formulación de estos conceptos y las leyes que los delimita, aplicando el método inductivo.

A pesar de estar afincada en la aproximación epistemológica positivista, los químicos orgánicos se mostraron reacios a admitir la fisicoquímica como disciplina. Aludían que no estaba fundamentada experimentalmente y que se basaba en meras elucubraciones teóricas. (Brock, 1998). Era comprensible, como se ha anotado, la formación matemática de estos especialistas no pasaba del álgebra (Schneer, 1975).

Si bien es con la formulación de la mecánica cuántica de matrices por W. Heisenberg (1901 - 1976) en 1925, y la ondulatoria de E. Schrödinger de 1926, que se empieza a cuestionar la mirada determinista, es posible afirmar que la discusión tiene sus inicios con el desarrollo de la termodinámica estadística, puesto que es a partir de ella que se inician las reflexiones, en términos probabilísticos, las interacciones entre los componentes de los objetos de conocimiento propuestos por termodinámicos y fisicoquímicos.

Si bien se alude a cada uno de los investigadores que se ocuparon de la introducción de la mirada termodinámica , como máquinas térmicas, no se asumen en esta reconstrucción como héroes (Fara, 2009). Una lectura entre líneas del contenido del presente artículo, permite estipular que este proceso fue fruto de una construcción colectiva (Kuhn, 1972), por cuanto para la época, el conocimiento circulaba en las revistas especializadas.

\section{Una conclusión intermedia}

Los colegas se preguntarán cómo las reconstrucciones realizadas en esta primera parte, tienen que ver con el objetivo propuesto de demostrar esa superación de la matemática algebraica dentro de la cual se construyó la química como ciencia y especialmente la química orgánica, por así decirlo, la disciplina reina dentro de la cual sus exponentes contribuyeron a que la actividad por ellos desempeñadas, adquiriera estatuto académico y, por tanto, ser considerada como una rama de la filosofía natural. 
Sin embargo y lo que se desea resaltar son dos hechos históricos, incluso con el empleo del término "hecho", que llama a malentendidos, que esa matematización requirió de la admisión del modelo cinético molecular de los gases y, en consecuencia, de una mirada necesaria de los procesos químicos, como sistemas termodinámicos. El tránsito no fue fácil, Aun cuando en las discusiones acerca de la conservación de la energía, había el preludio que desembocó en la necesidad curricular, de que la formación académica de los profesionales químicos, fueran más allá. La segunda parte del presente artículo se ocupará de los problemas didácticos.

\section{Referencias bibliográficas}

Bachelard, G. (1976). El materialismo racional. Buenos Aires: Paidos.

Bensaude - Vincent, B. y Stengers, I. (1997). Historia de la química. Madrid: Addison Wesley.

Bowler, P. J. y Morus, I. R. (2005). Panorama general de la ciencia moderna. Barcelona: Crítica.

Brock, W. H. (1998). Historia de la Química. Madrid: Alianza.

Caldin, E. F. (2002). The Structure of Chemistry In Relation to the Philosophy of Science. International Journal for Philosophy of Chemistry, Vol. 8, No. 2, $103-121$.

Del Re, G. (2000). Models and analogies in science. International Journal for Philosophy of Chemistry, Vol. 6, N0. 1, 5-15.

Fara, P. (2009). Breve historia de la ciencia. Madrid: Ariel.
Gallego Torres, A. P., Gallego Badillo, R. y Pérez Miranda, R. (2006). ¿Qué versión de ciencia se enseña en el aula? Sobre los modelos científicos y la didáctica de la modelación. Educación y Educadores, Vol. 9, No. 1, 1- 13.

Gallego Badillo, R., Pérez Miranda, R. y Gallego Torres A. P. (2009). Una aproximación histórico-epistemológica a las leyes fundamentales de la química. Revista Electrónica de Enseñanza de la Ciencia, Vol. 8, No. 1, Art. 19, $359-375$.

Ihde, A. J. (1984). The development of modern chemistry. New York: Dover.

Jensen, W. B. (1998a). Logic, history and the chemistry textbooks I: Does chemistry have a logical structure? Journal of Chemical Education, Vol. 75, No. 6, 679-687.

Jensen, W. B. (1998b). Logic, history and the chemistry textbooks II. Can we unmuddle the chemistry textbook? Journal of Chemical Education, Vol. 75, No. 7, 917 - 828.

Jensen, W. B. (1998c). Logic, history and the chemistry textbooks III: One chemical revolution or tree? Journal of Chemical Education, Vol. 75, No. 8, 961 - 969.

Kuhn, T. S. (1972). La estructura de las revoluciones científicas. México: Fondo de Cultura Económica.

Lakatos, I. (1983). La metodología de los programas de investigación. Madrid: Alianza.

Laín, P. y López, J. M. (1962). Panorama histórico de la ciencia moderna. Madrid: Guadarrama.

Lombardi, O. (1998). La noción de modelo en ciencias. Educación en Ciencias, Vol. II, No. $4,5-13$. 
Lévi - Strauss, C. (1964). El pensamiento salvaje. México: Fondo de Cultura Económica.

Lockemann, G. (1960). Historia de la Química. México: Uteha.

Mayr, E. (2006). Por qué es única la biología. Consideraciones sobre la autonomía de una disciplina científica. Buenos Aires: Katz.
Sánchez, J. S. (2009). El jardín de Newton. La ciencia a través de la historia. Barcelona: Crítica.

Schneer, C. J. (1975). Mente y materia. Barcelona: Bruguera.

Thuillier, P. (1975). La manipulación de la ciencia. Madrid: Fundamentos. 